Article

\title{
Viscoelastic Dampers for Rotors: Modeling and Validation at Component and System Level
}

\author{
Angelo Bonfitto * (D), Andrea Tonoli (1) and Nicola Amati \\ Department of Mechanical and Aerospace Engineering, Polytechnic University of Turin, 10129 Turin, Italy; \\ andrea.tonoli@polito.it (A.T.); nicola.amati@polito.it (N.A.) \\ * Correspondence: angelo.bonfitto@polito.it; Tel.: +39-011-090-6240
}

Received: 20 October 2017; Accepted: 13 November 2017; Published: 16 November 2017

Featured Application: The presented methodology is currently adopted in production lines for identification and testing procedures on viscoelastic dampers for turbomolecular pumps.

\begin{abstract}
The aim of this paper is to propose a methodology for the modelling, testing, and the parameter identification of viscoelastic supports for rotating machines at the component and system level. Although the use of this type of dampers is common in rotordynamic applications, the strict dependence on the working frequency of the material parameters makes their behavior hard to predict and recommends grounding the design on experimental data of the characteristics. A dedicated test rig is adopted to characterize the supports and validate the modelling approach at component level. A parameter identification procedure is carried out from the experimental results to extract the mechanical properties of the supports. To this end, a Genetic Algorithm (GA) is adopted to search the most fitting values of damping and stiffness of the reference model. The choice of GA is motivated by the need of adopting a technique that can be easily implemented on industrial control units, being that the dedicated machine is supposed to be used in production lines for testing procedures. The results are obtained in terms of complex stiffness as a function of the frequency and are then translated in classical stiffness and damping components that are commonly used in rotordynamics. Afterwards, they are introduced in the model of a turbo-molecular pump adopted as a case study to evaluate the correctness of the proposed methodology at system level at stand-still and with the pump in rotation. The proposed approach is effective, showing a good match between the numerical model and the experimental results even with a relatively low order model of the viscoelastic supports and on a complex rotordynamic system.
\end{abstract}

Keywords: vibration damping; viscoelasticity; rotordynamics; Genetic Algorithm; identification; turbomolecular; dampers

\section{Introduction}

Vibrations in mechanical structures are typically caused by structural issues like effects of the material fatigue damage and wear, and are the cause of loss of performance and reliability. In rotordynamics, these negative consequences are emphasized and strongly influence and constrain most of the design phases. Vibrations in rotating machines can be induced by the unavoidable presence of unbalance giving rise to synchronous vibrations in the vicinity of critical speeds. This phenomenon is strictly dependent on the quality of balancing, spin speed, and the presence of damping. It imposes implementing vibration control techniques that can be chosen among a wide range of technologies, according to the application requirements. In aerospace, the adoption of squeeze film dampers (SFD) is common practice. They are very effective in reducing the response for the moderate range of residual unbalance expected from the rotor balancing process and are self-sufficient and robust in the event of 
high unbalance levels [1]. However, their performance is affected by vibration frequency, engine oil temperature, supply pressure, and thermal growth of the bearing housing. In the oil and gas industry, and in aerospace, solutions based on active/semi-active hydraulic systems, electrorheological [1,2], and magnetorheological [2] solutions have been proposed for the adaptation of the damping force to the operating conditions. However, these devices cannot avoid drawbacks related to the aging of the fluid and to the tuning that is required for compensating the temperature and frequency effects. Among other technologies, magnetic bearings and dampers witnessed a steady growth in applications, like vacuum pumps, compressors for air conditioning, turbo-compressors in oil and gas industry, turbines for energy recovery, and storage, such as kinetic energy storage systems (KESS) and compressed air energy storage (CAES) [3-6]. They allow for contactless operation and lack the need of lubrication and oil supply systems. Although attractive, this technology still presents shortcomings related to size and costs. Viscoelastic dampers represent an effective and low-cost solution in high speed applications like turbo-molecular pumps and machine tool spindles or in industrial sectors like automotive, where the construction of engine mounts, torsional dampers and shock absorbers is based on the adoption of these components. Nevertheless, although compact and efficient, the frequency and temperature dependency of the material parameters makes the rotordynamic behavior of the machines equipped with viscoelastic supports hard to predict at design stage [7]. Currently, the design of elastomeric dampers is mainly based on a trial-and-error approach, but a more rigorous method is often required to know exactly the behavior of the supports in operating conditions and to achieve design refinement and performance optimization in terms of vibrations amplitude, unbalance response reduction, and critical speeds tuning.

The objective of this paper is to propose a methodology to identify the behavior of viscoelastic dampers and to predict the rotordynamic behavior of shafts supported by them. The technique is based on a preliminary experimental characterization at the component level of the supports by means of a dedicated test rig allowing the replication of the unbalance synchronous excitation by means of two orthogonal actuators. The transfer functions measured between the force of the actuator and the traction or compression generated on the elastomer permits to obtain the behavior of the supports along the whole spin speed range in the form of complex stiffness. Afterwards, the parameters of the reference model adopted to represent the dampers are identified, according to the experimental results by means of a Genetic Algorithm (GA). The choice of GA is motivated by the need of adopting an identification technique that can be easily implemented in industrial electronic control units, since the dedicated machine is supposed to be adopted in the production line for testing operations. In the authors' knowledge, the adoption of GA for the identification of viscoelastic parameters is not yet reported in literature. The designed GA is based on the survival principle of the fittest and aims to search the optimal parameters by minimizing a fitness function that is expressed as the weighted sum of the Mean Square Error (MSE) between the magnitude and phase of the numerical and experimental transfer functions. Three different selection methods are proposed and compared: Normalized Geometric, Tournament, and Roulette Wheel Selection. All of the procedures converge to the same values and the parameters from Normalized Geometric Selection are chosen and translated in classical stiffness and damping components to be useful for the rotordynamic model of assembled rotors. The approach at component level is effective since a good match between the numerical model and the experimental results is shown, even with a relatively low order model of the viscoelastic supports.

The methodology is then validated at system level in a real application that is represented by a turbo-molecular pump, a type of multi-stage vacuum machine. The two dampers identified in the first section are the same installed in the pump. The resulting model of the dampers is introduced in the full model of the pump as a connection between the free-free models of the rotor and of the stator. The validation of the proposed approach is performed by impact tests at null speed and by the measurement of the unbalance response. The close correlation between model response and experimental data at standstill, and with the shaft at full speed, demonstrates the effectiveness of the approach also at system level. 


\section{Viscoelastic Model Identification}

\subsection{Machine for Testing Operations}

In rotordynamics, the adoption of elastomeric dampers interposed between the bearings and the non-rotating part of the machine is a common practice $[8,9]$.

Two different layouts of the suspension can be adopted, as illustrated in Figure 1. The first (a) exploits a viscoelastic ring (5) press-fitted directly between the stator (1) and the external part of the ball bearing (4), while in the second one (b), the viscoelastic material (5) is molded between two metallic supports (2), the external is screwed on the stator while the internal is press-fitted on the ball bearing. In the first configuration, the friction between the rubber and the bearing modifies the axial preload of the bearing in an uncontrolled way, and hence the radial stiffness of the suspension. In the second configuration, this problem is not present since no relative displacement between the viscoelastic element (5) and its metallic supports (2) occurs. The preload of the bearing can be provided in a predictable way by installing the two metallic supports of the rubber with a small misalignment along the axial direction of the suspension. The configuration installed in the machine that is considered in this paper exploits the layout (b).

a)
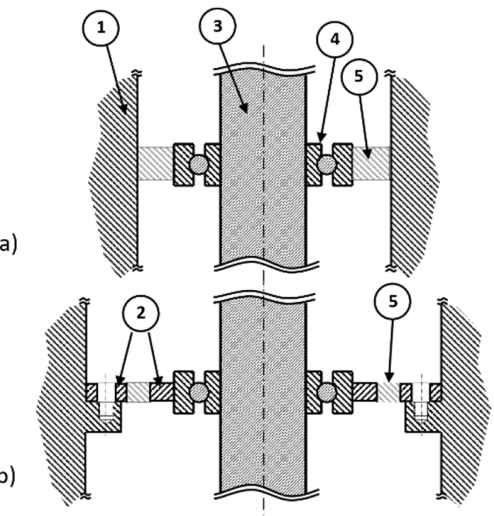

Figure 1. Layout of the suspension. (a) Viscoelastic element (square-ring) press-fitted directly between the stator and the ball bearing. (b) Viscoelastic element molded between two annular elements. The internal element is press-fitted on the rotor, while the external one is screwed on the stator. (1) Stator of the machine. (2) Damper metallic supports. (3) Shaft. (4) Ball bearing. (5) Viscoelastic section.

Although the presented solution appears simple and reliable, the viscoelastic nature of elastomers imposes to perform experimental identification of the damper to predict its behavior at design stage at varying operation frequencies and applied static loads. To this end, a dedicated machine (Figure 2) has been designed and realized to simulate the forces acting on rotors in working conditions, providing profiles simulating the synchronous excitation due to the unbalance.
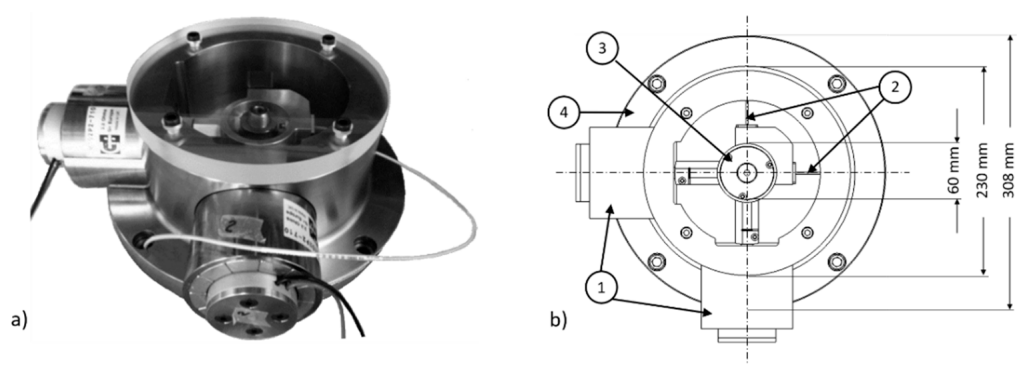

Figure 2. (a) Picture of the machine. (b) View from top. (1) Actuators. (2) Displacement sensors. (3) Damper support. (4) Body of the machine. 
A couple of orthogonal linear voice coils allow to provide a rotating force vector simply driving the actuators in order to generate two sinusoidal forces with the same amplitude and the same frequency, but with a phase-lag of 90 degrees between them. In principle, this layout permits to also perform a linear characterization if one-directional measures are requested. In addition to being used in the design phase to characterize elastomer mechanical parameters at the component level, the rig, whose maximum excitation frequency is $1.5 \mathrm{kHz}$ equivalent to a spin speed of $90,000 \mathrm{rpm}$, can be exploited to perform tests concerning the reliability and durability of the supports in working conditions.

The machine is designed to be installed on a seismic mass ((10) in Figure 3). The main steel structure is composed of an external body (9) on which the static parts of the actuators (3) are installed. The mobile part of the voice coils (1) is connected to the external support of the damper (11) with a screw (4), and is supported by a collet (2) to guarantee a good alignment of the actuation direction. The internal support of the damper (12) is screwed in the main body. Two eddy current displacement sensors (14) are adopted to detect the relative movement between the internal and the external part of the damper in order to evaluate its traction and compression.

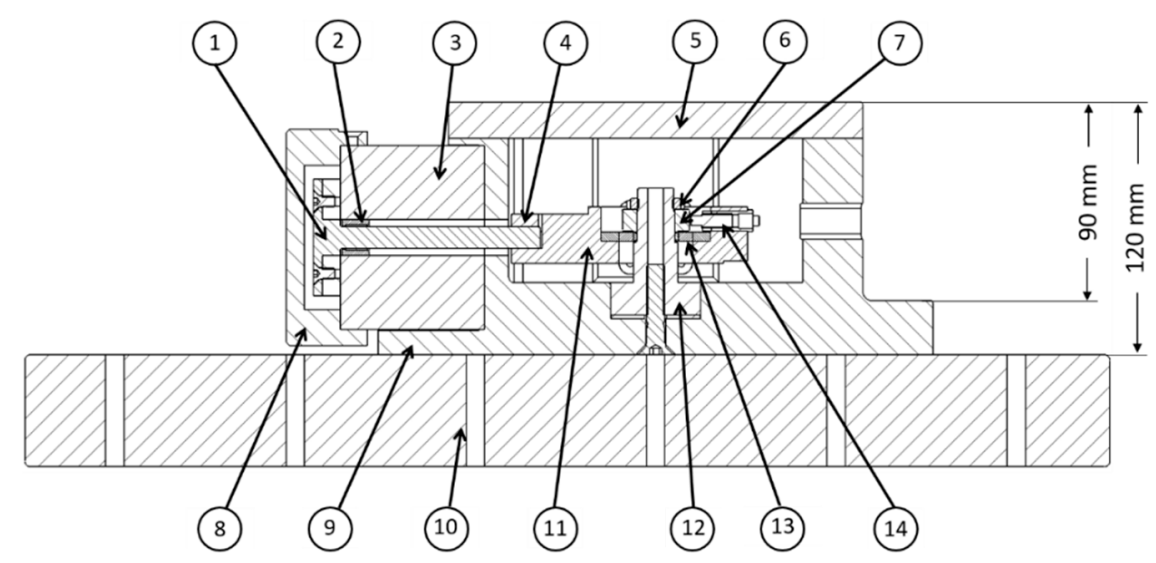

Figure 3. Cross-section of the machine from lateral view. (1) Mobile part of the actuator. (2) Collet for actuator support. (3) Static part of the actuator. (4) Screw to fix actuator to the machine. (5) Plexiglass cover. (6) Top nut. (7) Metallic spacer. (8) Metallic cover of the actuator. (9) Body of the machine. (10) Seismic mass. (11) External support of the damper. (12) Internal support of the damper tower.

(13) Viscoelastic damper. (14) Displacement sensor.

The two actuators, whose current-force coefficient is $37 \mathrm{~N} / \mathrm{A}$, can exert a maximum force of $200 \mathrm{~N}$ with an excitation frequency ranging from 0 to $1500 \mathrm{~Hz}$. They are supplied with a DC (Direct Current) voltage of $30 \mathrm{~V}$ at a maximum current of $5 \mathrm{~A}$. The maximum displacement that is guaranteed on the whole frequency range is $10 \mu \mathrm{m}$. Parameter settings, result displaying, and data logging are realized by means of an HMI (Human Machine Interface) running on a PC in CAN (Controller Area Network) communication with the electronic drivers of the plant. The machine is adopted for testing procedures in the production lines of the dampers.

\subsection{Reference Model for Parametric Identification}

Ideal elastic materials do not dissipate energy when deformed and can be represented with only a linear spring (a in Figure 4). However, since no actual material is ideal elastic, the simplest way to model material damping is to assume that the material reacts with a force depending only on the strain rate. These two basic behaviors can be combined to obtain a more accurate representation of the material exploiting a simple series of a spring and a damper (b) or by means of more elaborate spring-damper models like Kelvin's (c) and Wiechert's (d) representation. 


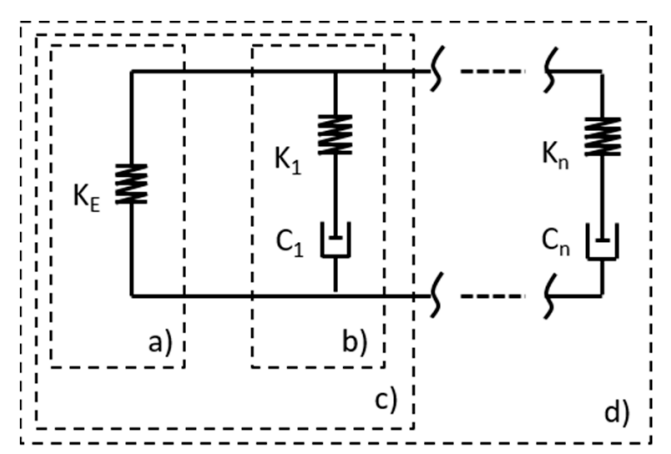

Figure 4. Most common representations of viscoelastic material. (a) Ideal elastic material (Hooke's representation); (b) Combination of restoring and damping force (Maxwell's representation);

(c) Kelvin's representation; and, (d) Wiechert's representation.

These models represent the equilibrium restoring stiffness with an isolated spring $K_{E}$ in parallel with one (c) or several (d) Maxwell's arms.

In the case of configuration (c), the force provided by the elastomeric material is obtained as the sum of the force in the Maxwell arm and that in the isolated spring

$$
F=F_{M}+F_{E}
$$

The force in the Maxwell arm in Laplace domain is depending on the traction/compression $x$ applied to the material and is equal to:

$$
F_{M}=\frac{k_{1} s}{s+\frac{k_{1}}{C_{1}}} x
$$

Adding the force $F_{E}=k_{E} x$ in the equilibrium spring, the total force is

$$
F=k_{E} x+\frac{k_{1} s}{s+\frac{k_{1}}{C_{1}}} x=\left\{k_{E}+\frac{k_{1} s}{s+\frac{k_{1}}{C_{1}}}\right\} x
$$

This modeling approach can be extended to the case of Wiechert's representation (d), where the total force $F$ is the sum of the components of each parallel arm

$$
F=F_{E}+\sum_{j} F_{j}
$$

Extending from Equation (2),

$$
F_{j}=\frac{k_{j} s}{s+\frac{k_{j}}{c_{j}}} x
$$

and

$$
F=F_{E}+\sum_{j} F_{j}=\left\{k_{E}+\sum_{j} \frac{k_{j} s}{\left(s+\frac{k_{j}}{c_{j}}\right)}\right\} x
$$

follows. In this paper, Kelvin's model is adopted to represent the behavior of the dampers under test effectively. However, the proposed methodology is valid also with Wiechert's representation should it be necessary to take relaxation time distribution into account. 
The characterization of the viscoelastic dampers under test is based on the following representation of the system

$$
H=\frac{F_{A C T}}{x}=m s^{2}+\left(k_{E}+\frac{k_{1} s}{s+\frac{k_{1}}{C_{1}}}\right)+\frac{K_{A C T} S}{s+\frac{K_{A C T}}{C_{A C T}}},
$$

where $H$ is the transfer function between the traction/compression $x$ of the elastomer, and the force $F_{A C T}$ applied on it, $m$ is the inertial action obtained as the sum of the mass of the damper support ((11) in Figure 3) and of the mobile part of the actuator (1). The second component in Equation (7) is the representation of the damper, while the third part models the losses on the actuator due to eddy currents generation, which is represented by a dynamic identified with FEM (Finite Element Method) computation. This has been performed with the software COMSOL Multiphysics 4.3a (Comsol Inc., Stockolm, Sweden, May 2012) following the indications reported in [10], where it is demonstrated that the mechanical impedance of an eddy current damper can be represented as a series of a viscous damper and a spring.

A sensitivity analysis to evaluate the effects of the change of the parameters $K_{E}, K_{1}$, and $C_{1}$ on the damper dynamic and consequently on the measured transfer function is illustrated in Figure 5. The plots on the left side $(\mathrm{a}-\mathrm{c})$ report the trend of Equation (3), while the corresponding effects on Equation (7) are shown on the right side (d-f). It is evident from (d) and (e) that the inertial effects of mass $m$, representing the mobile part of the voice coil and the mechanical components connected to the voice coil stem, is dominant in $F_{A C T} / x$ at high frequencies with respect to other parameters.
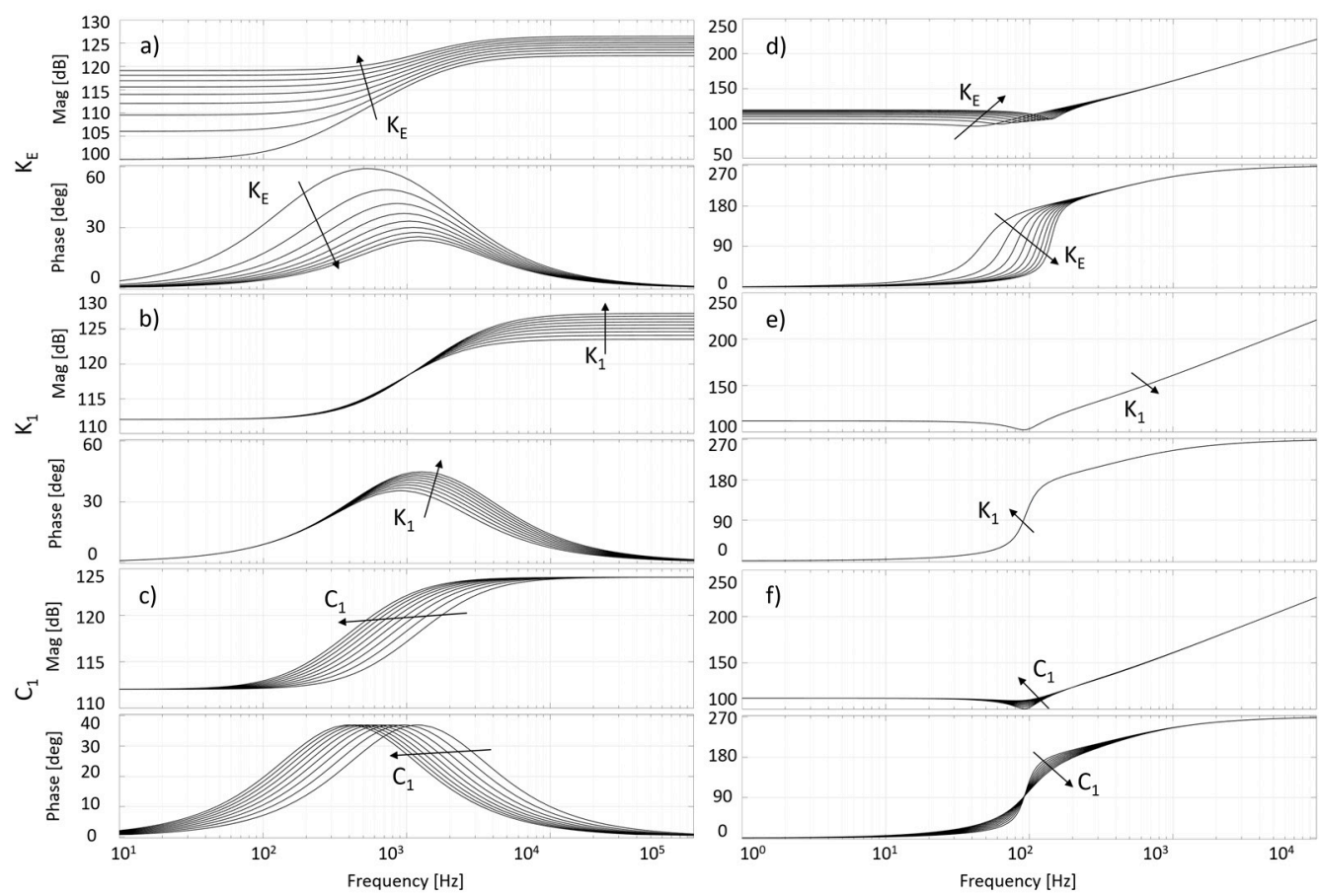

Figure 5. Sensitivity analysis on support parameters when considering Kelvin's model. Left side (a-c): damper behavior as in Equation (3). Right side (d-f): $F_{A C T} / x$ behavior as in Equation (7). (a) Damper dynamic at varying $K_{E}$. (b) Damper dynamic at varying $K_{1}$. (c) Damper dynamic at varying $C_{1}$. (d) $F_{A C T} / x$ at varying $K_{E}$. (e) $F_{A C T} / x$ at varying $K_{1}$. (f) $F_{A C T} / x$ at varying $C_{1}$. 


\subsection{Experimental Characterization and Identification by Genetic Algorithm}

The proposed procedure is tested on the two different dampers that have been installed in the compressor side (CS) and in the shaft end side (SES) of the turbo-molecular pump that is used for the experimental validation at the system level, as reported in the final section of this paper.

The transfer function $H$ (Equation (7)) is measured experimentally $\left(H_{E X P}\right)$ with a sweep test from 0 to $1.5 \mathrm{kHz}$, and is then compared to the numerical equivalent $\left(H_{N U M}\right)$ to identify the values of $K_{E}$, $K_{1}$, and $C_{1}$. The identification is performed automatically on the DSP (Digital Signal Processor) by means of a Genetic Algorithm (GA), aiming to minimize the difference between the numerical $\left(H_{N U M}\right)$ and the experimental $\left(H_{E X P}\right)$ results.

GAs are search and optimization techniques that are inspired by two biological principles, namely the process of natural selection and the mechanics of natural genetics. They consist of a mechanism of selective reproduction acting on a set of potential solutions, called chromosomes, whose correctness is measured by means of a fitness function. The collection of potential solutions, called population, is manipulated to perform a search based on the survival principle of the fittest. The chromosomes are the encoded representations of all the parameters of the solution. Each chromosome is compared to the others in the population and evaluated with a fitness rating that indicates how successful the solution is. The first population is created randomly while the following are generated to encode better solutions using genetic operators or evolution operators, such as elitism, crossover, and mutation. Elitism allows for saving the best chromosomes before reproduction, crossover, and mutation, enabling the new reproduction by either merging the chromosomes in the current population or by modifying some of them. Several algorithms of crossover and mutation are present in the literature, in the present case the Arithmetic Crossover Procedure and the Uniform Mutation Method are adopted. The selection of the best individuals for each generation can be performed with different methods, in this case, three of them are tested and compared: Normalized Geometric Selection (NGS), Tournament Selection (TS), and Roulette Wheel Selection (RWS). The creation of new generations stops when a fitness target is reached or after a certain number of reproductions [11-13].

In the proposed GA, each chromosome is encoded as a vector of real numbers of the same length as the solution vector. The parameters to be optimized are $K_{1}$ and $C_{1}$, therefore the chromosome $j$ of generation $i$ is represented as

$$
C_{j}^{i}=\left[c_{j 1}^{i}, c_{j 2}^{i}\right]
$$

where $c_{j 1}=K_{1}$ and $c_{j 2}=C_{1}$.

The upper and lower bounds of the two parameters for both the characterized dampers are

$$
\begin{aligned}
& c_{j 1, \text { min }}=1 \times 10^{5} \mathrm{~N} / \mathrm{m}, \\
& c_{j 1, \text { max }}=4 \times 10^{6} \mathrm{~N} / \mathrm{m}, \\
& c_{j 2, \min }=100 \mathrm{Ns} / \mathrm{m}, \\
& c_{j 2, \text { max }}=400 \mathrm{Ns} / \mathrm{m} .
\end{aligned}
$$

The fitness function $F_{o b j}$ is established to provide a performance measure of the generated chromosomes. The difference between analytical $\left(H_{N U M}\right)$ and experimental $\left(H_{E X P}\right)$ results is considered as the error $e$ to be minimized by means of Mean Square Error (MSE) computation. Both magnitude (Mag) and phase (Pha) are considered according to the fitness function

$$
F_{o b j}=1 /\left(W_{m} \frac{\sum_{q=1}^{n}\left(\operatorname{Mag}\left(H_{N U M, q}\right)-\operatorname{Mag}\left(H_{E X P, q}\right)\right)^{2}}{n}+W_{p} \frac{\sum_{q=1}^{n}\left(\operatorname{Pha}\left(H_{N U M, q}\right)-P h a\left(H_{E X P, q}\right)\right)^{2}}{n}\right)
$$

where $n$ is the size of the frequency vector, and $W_{m}$ and $W_{p}$ are the weighting coefficients for magnitude and phase MSE, respectively, both set to 0.5 in this case. The GA is implemented exploiting a population of 60 individuals in 60 generations according to the following procedure:

1. Computation of $K_{E}$ from Equation (7) at steady-state $(s=0)$. 
2. Random generation of the initial population of the parameters $K_{1}$ and $C_{1}$.

3. Computation of the fitness value for each chromosome by means of $F_{o b j}$.

4. Selection process with the NGS, TS (with 100 rounds) and RWS procedure to select the best set of individuals.

5. Application of the elitist selection method to avoid the loss of best chromosomes during the creation of the new population. Elitism allows for saving a priori best individuals before the production of the next generation.

6. Execution of the Arithmetic Crossover Procedure to generate new individuals.

7. Execution of the Uniform Mutation Method to perturb the population with an occasional random alteration to avoid sub-optimal convergence of the solution.

8. Going back to step 3 of this procedure and evaluation of the new population fitness value according to $F_{o b j}$. The termination of the iteration occurs at the production of the 60th generation.

One occurrence of the best population of the optimized parameters is reported in Figure 6 for the two dampers. The three selection procedures are compared (NGS in solid line, TS in dashed line, and RWS in dotted line) showing the convergence to the same final values with a good level of robustness of the optimization method. NGS is adopted because of the slightly lower execution time. However, the other two methods can be adopted without the loss of performance. The identified parameters are

$$
\begin{array}{ll}
K_{1_{S E S}}=1.1034 \times 10^{5}, & C_{1_{\text {SES }}}=8.2923 \times 10^{2}, \\
K_{1_{C S}}=8.9207 \times 10^{5}, & C_{1_{C S}}=1.1615 \times 10^{3} .
\end{array}
$$

The comparison between $H_{N U M}$ and $H_{E X P}$ is shown in Figure 7 . The noise measured at frequencies higher than $1 \mathrm{kHz}$ is due to bad measurement but it can be neglected since it is out of the range of interest. Indeed, the nominal speed of the pump where the dampers are supposed to be installed is $48,000 \mathrm{rpm}$. The values of magnitude and phase at frequency higher than $1 \mathrm{kHz}$ have not been taken into account in the GA identification.

The frequency response of the damper $\left(G_{D}\right)$ is then obtained as

$$
G_{D}=k_{E}+\frac{k_{1} s}{s+\frac{k_{1}}{C_{1}}}=\frac{F_{A C T}}{x}-m s^{2}-\frac{s K_{A C T}}{s+\frac{K_{A C T}}{C_{A C T}}} .
$$

In the final part of the identification, the dynamic of the damper is obtained from Equation (12) to be usable for rotordynamic models. Two contributes are identified in the frequency range of operations: in-phase or storage stiffness $\left(K_{P}\right)$ and in-quadrature or loss stiffness $\left(K_{Q}\right)$, obtained as follows:

$$
\begin{aligned}
& K_{P}=\operatorname{Re}\left(G_{D}\right), \\
& K_{Q}=\operatorname{Im}\left(G_{D}\right) .
\end{aligned}
$$

Figure 8 reports the trends of $K_{P}$ and $K_{Q}$ of the SES (a) and CS (b) dampers of the pump. It is possible to obtain equivalent viscous damping $\left(C_{e q}\right)$ of the support with

$$
C_{e q}=\frac{1}{\omega} K_{Q}
$$

where $\omega$ is the actuator excitation frequency in $\mathrm{rad} / \mathrm{s}$. 
a)
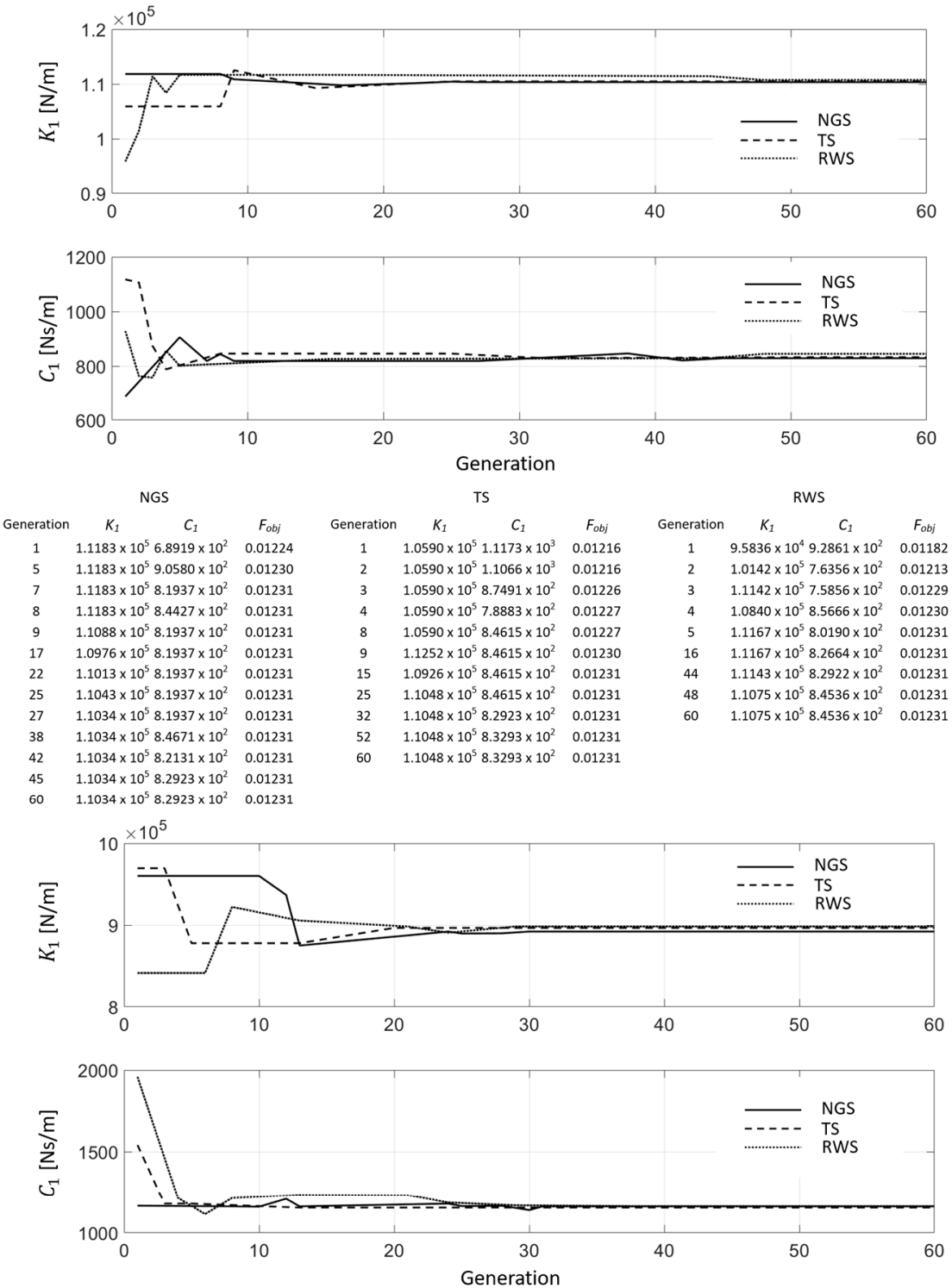

\begin{tabular}{cccc}
\multicolumn{4}{c}{ NGS } \\
Generation & $K_{1}$ & $C_{1}$ & $F_{o b j}$ \\
1 & $9.6011 \times 10^{5}$ & $1.1662 \times 10^{3}$ & 0.03413 \\
8 & $9.6011 \times 10^{5} 1.1619 \times 10^{3}$ & 0.03415 \\
10 & $9.6011 \times 10^{5} 1.1600 \times 10^{3}$ & 0.03415 \\
12 & $9.3689 \times 10^{5} 1.2087 \times 10^{3}$ & 0.03637 \\
13 & $8.7499 \times 10^{5} 1.1619 \times 10^{3}$ & 0.0375 \\
24 & $8.9207 \times 10^{5} 1.1790 \times 10^{3}$ & 0.03850 \\
25 & $8.8971 \times 10^{5} 1.1636 \times 10^{3}$ & 0.0385 \\
28 & $8.8994 \times 10^{5} 1.1650 \times 10^{3}$ & 0.03851 \\
30 & $8.9207 \times 10^{5} 1.1395 \times 10^{3}$ & 0.0385 \\
31 & $8.9207 \times 10^{5} 1.1615 \times 10^{3}$ & 0.03860 \\
60 & $8.9207 \times 10^{5} 1.1615 \times 10^{3}$ & 0.03860
\end{tabular}
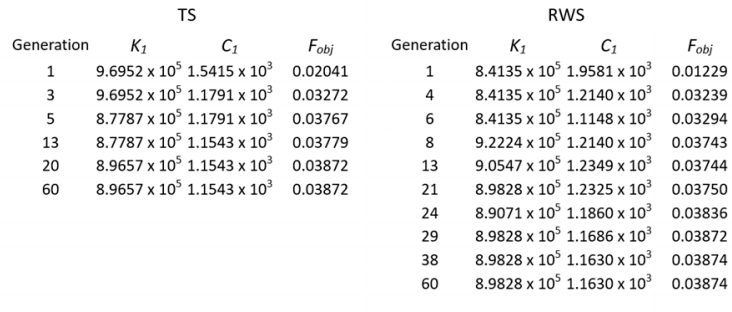

Figure 6. One occurrence of the best population of shaft end side (SES) (a) and compressor side (CS) (b) damper parameters computed with Normalized Geometric Selection (NGS) (solid line), Tournament Selection (TS) (dashed line) and Roulette Wheel Selection (RWS) (dotted line). The tables report the convergence trend of the best chromosome and their fitness value for each method. 


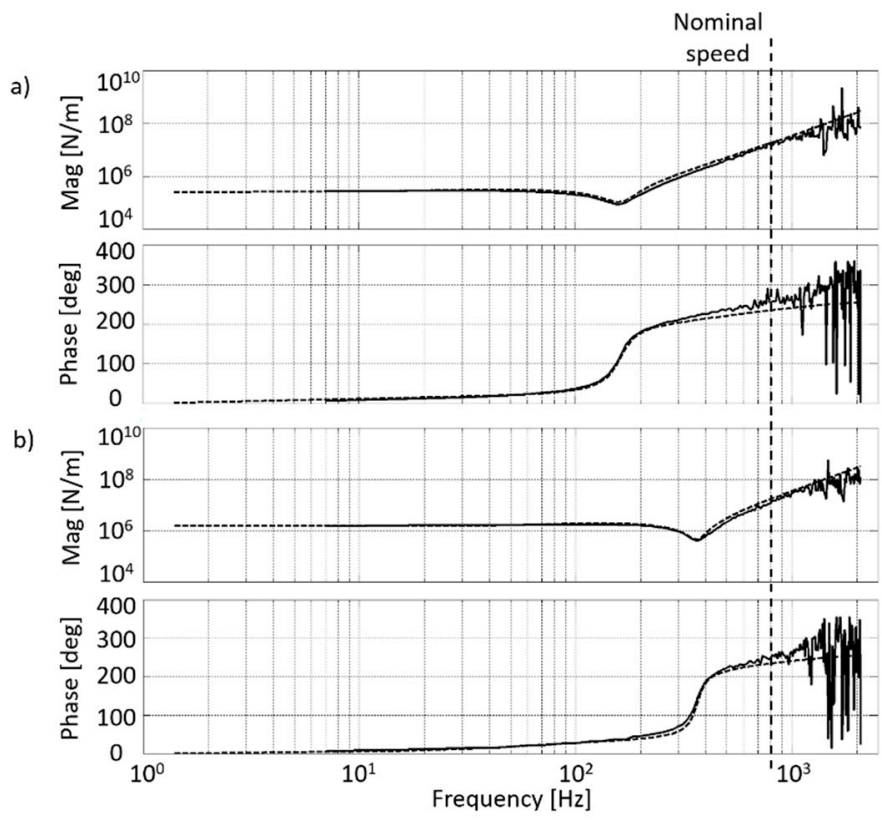

Figure 7. Numerical $H_{N U M}$ (dashed line) and experimental $H_{E X P}$ (solid line) behavior of the damper. (a) SES support of the turbo-molecular pump. (b) CS support of the turbo-molecular pump.

a)

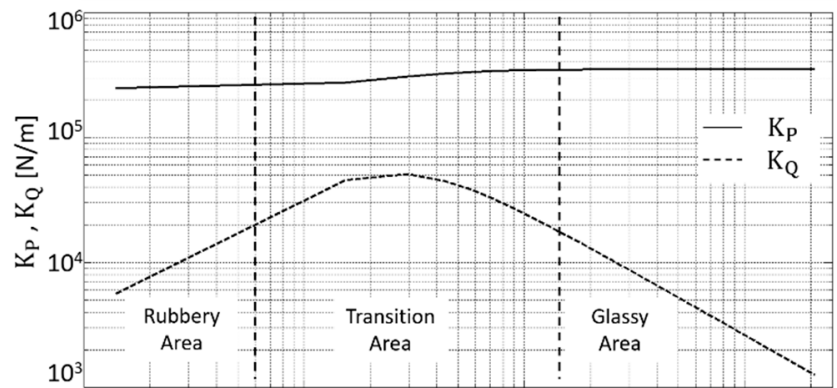

b)

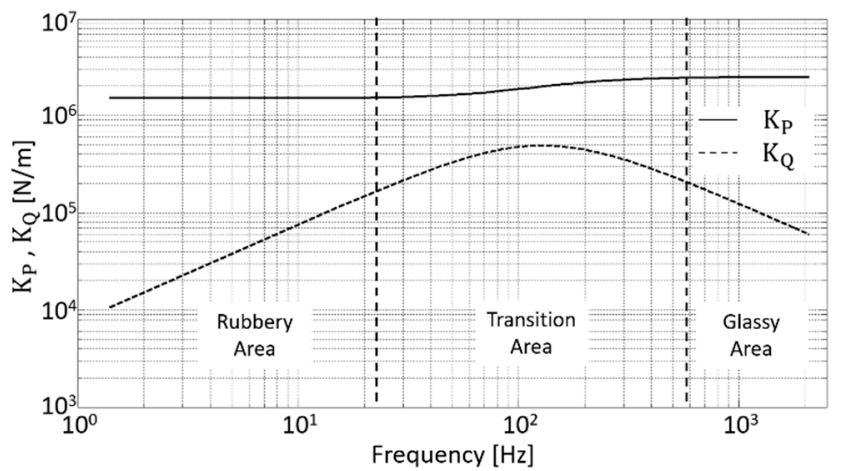

Figure 8. Dynamic behavior of the damper usable in rotordynamic models. In-phase stiffness $K_{P}$ (solid line). In-quadrature stiffness $K_{Q}$ (dash-dotted line). (a) SES support of the turbo-molecular pump. (b) CS support of the turbo-molecular pump.

The elastomeric support provides a low value of stiffness and damping at low frequencies. The maximum contribution of damping is recorded in the central area where the stiffness is rising. At high frequencies, the stiffness converges to a maximum value, while the damping is steadily decreasing.

In the systems that are equipped with viscoelastic supports, the goal is to make the transition area of the damper correspond with the natural frequencies of the structure in order to exploit the support where its damping action reaches its maximum. This objective is pursued by properly designing the 
geometry of the damper to regulate the in-phase stiffness and the maximum admitted displacement and by selecting the appropriate composition of the viscoelastic material, which is more related to damping, to tune the shape of the in-quadrature stiffness. For what the case presented in this paper is concerned, the two tested dampers have been designed to make the transition area correspond with the critical speeds of the rotor, as verified in the experimental results of the section dedicated to the validation at system level.

The results of the presented identification procedure have been obtained at a temperature of $25^{\circ} \mathrm{C}$. Nevertheless, as abovementioned, the mechanical properties of elastomeric materials are markedly influenced also by temperature. The temperature dependent characterization of the viscoelastic dampers is out of the goals of this paper. Nevertheless, it is possible to also achieve this kind of identification by carrying out the same proposed procedure in the whole temperature range of interest. As an alternative, since the effects of an increase of frequency or a decrease of temperature have similar effects on viscoelastic material behavior, it is possible to obtain the curves that are related to changes in temperature at constant frequency from those that are related to frequency at constant temperature and vice versa $[14,15]$.

\section{Rotordynamic Validation for the Identified Damper Models}

The final part of the study is focused on the validation of the approach at system level. To this end, the two dampers previously characterized are installed in a turbo-molecular pump to perform the experimental characterization. The machine adopted has a pumping capacity of $1.2 \mathrm{~L} / \mathrm{s}$, the weight of the rotor is $0.9 \mathrm{~kg}$, and the nominal speed is $48,000 \mathrm{rpm}$. The whole system is represented by means of an FEM model of the rotor and stator that is connected through the two dampers. The rotordynamic model has been realized by means of a FE (Finit Element) code (Dynrot), representing the rotating and the static parts with Timoshenko beam elements. Figure 9 shows the main nodes of the mesh along the shaft and the stator; (1) represents the stator that in the left-hand side has been considered as an unstructured mass on the left-hand side whose stiffness contribute is null (dotted part); (2) is the rotor whose pumping part is replaced by a mock-up provided with two discs to replicate the inertial behavior of the original bladed impeller; (3); and, (4) are the bearings and viscoelastic supports on the CS and on the SES, respectively. Gyroscopic effects are taken into account in all of the elements and rotating masses.

The dynamic equation of the rotating parts in the rotor reference frame is

$$
\mathbf{M} \ddot{q}(t)+(\mathbf{C}+\mathbf{G}) \dot{q}(t)+(\mathbf{K}+\mathbf{H}) q(t)=F_{u}
$$

where $q(t)$ includes the generalized displacements, $\mathbf{M}$ is the symmetric mass matrix, $\mathbf{C}$ and $\mathbf{G}$ are the symmetric damping and the skew-symmetric gyroscopic matrices, $\mathbf{K}$ and $\mathbf{H}$ are the symmetric stiffness and the skew-symmetric circulatory matrices, and $F_{u}$ is the unbalance force [16].

The experimental tests have been conducted in three steps: test on the shaft in free-free condition to validate its rotordynamic model (A); test in static conditions (B) and in rotation (C) on the assembled pump including rotor, stator and dampers.

(A) The rotor model is validated by means of impact tests on the free-free rotor. The transfer functions between the force (input) and the acceleration (output) are measured (Figure 10). The force is provided with an instrumented hammer in correspondence of the higher part of the impeller mock-up, while the acceleration is measured in the lower part of the shaft in correspondence of the CS (a) and SES (b) damper location. Solid and dashed lines report the experimental results and the numerical computations, respectively. 
a)

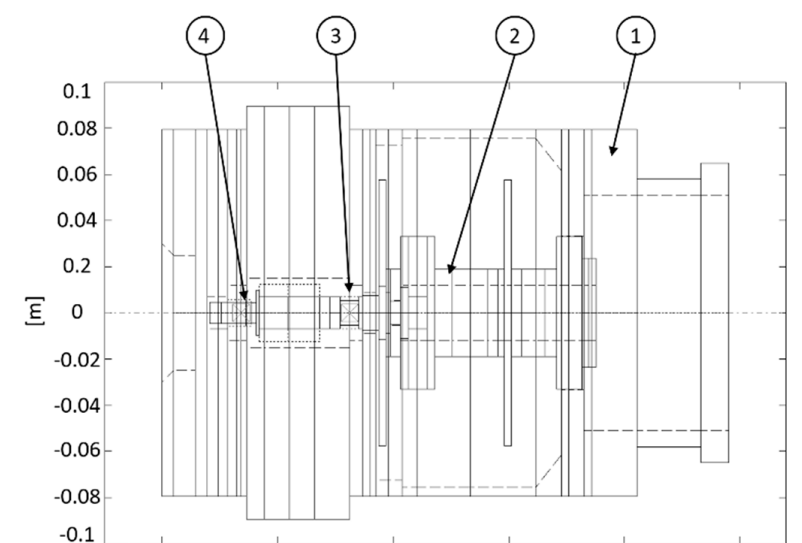

b)

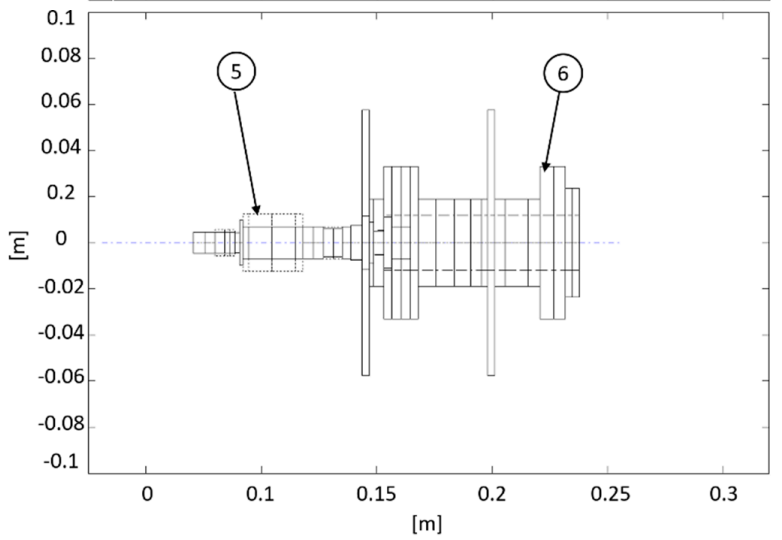

Figure 9. Turbo-molecular pump discretization for Finite Element Method (FEM) rotordynamic analysis. (a) Complete system. (b) Shaft. (1) Stator. (2) Rotor. (3) Bearing and viscoelastic support on the compressor side (CS). (4) Bearing and viscoelastic support on the shaft end side (SES). (5) Rotating part of the electric motor. (6) Impeller mock-up.

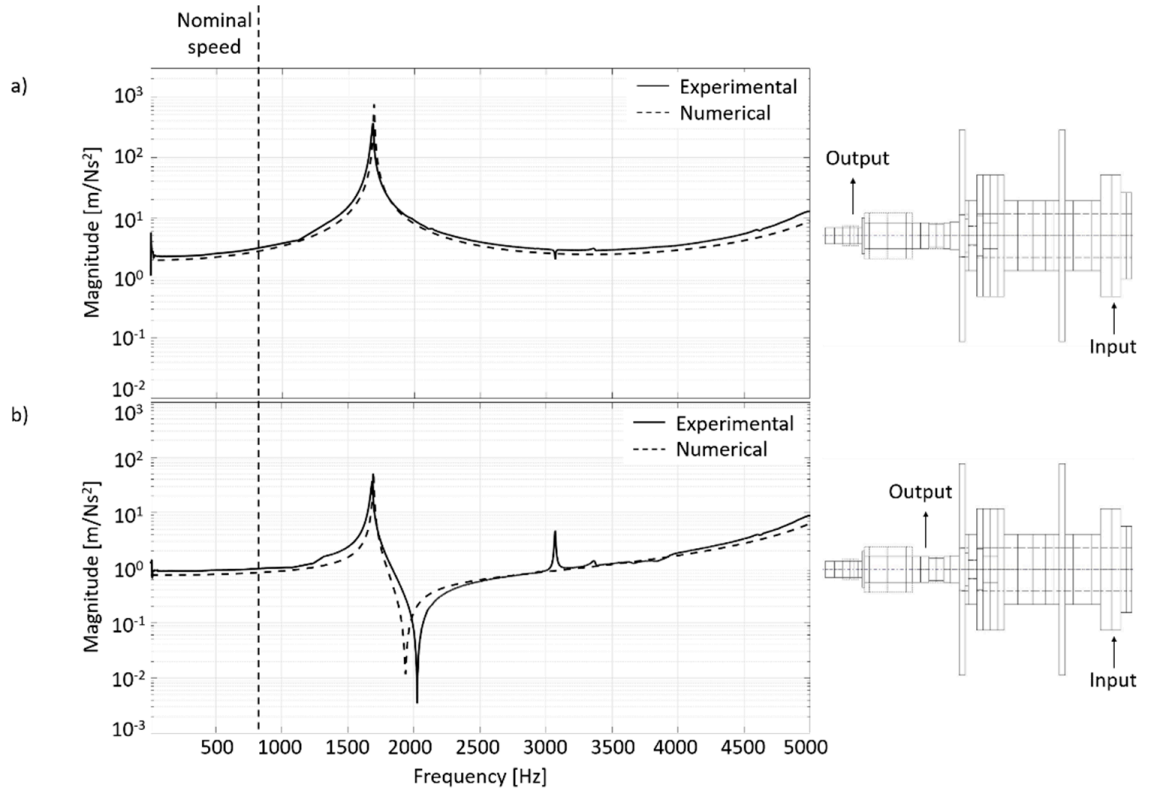

Figure 10. Rotor characterization. Transfer functions measured between the force on the higher part of the impeller mock-up (input) and the acceleration in correspondence of the SES (a) and CS (b) damper locations. 
(B) The assembled pump that is equipped with the two characterized dampers is tested in static condition with impact tests to obtain the transfer functions between the force (input) and the acceleration/displacement (output) (Figure 11). The force is provided with an instrumented hammer in correspondence of the higher part of the stator. The acceleration is measured on the stator (a) and on the rotor $(b-d)$. The relative displacement between the rotor and the stator is obtained with capacitive sensors (e,f). Solid and dashed lines are experimental and numerical results, respectively. The natural frequencies in the range of interest are located at $83 \mathrm{~Hz}$ and $472 \mathrm{~Hz}$, where both dampers are in the transition area and can provide damping, as illustrated in Figure 8.

The proposed modeling and identification methodology can be indifferently applied to rigid and flexible rotors. In this paper, the more complete approach for flexible rotors is illustrated. However, the pump that is used for the experimental validation is designed to work in subcritical condition with respect to flexible modes, thus it could be represented with a rigid body model without the loss of information.

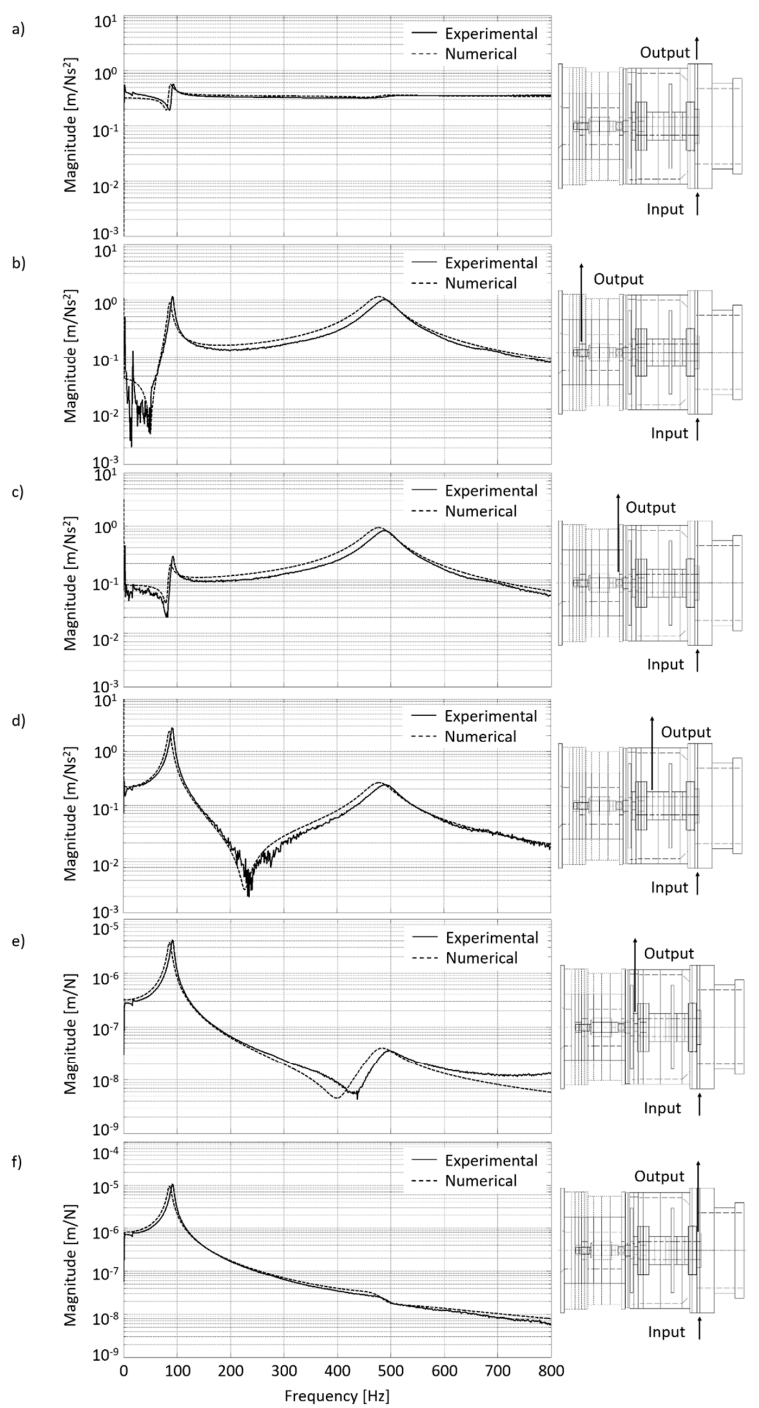

Figure 11. Characterization in static conditions of the assembled pump by impact test. The input force is on the stator while the measured outputs are: (a) Acceleration of the stator. (b) Acceleration of the rotor in correspondence of the SES damper location. (c) Acceleration of the rotor in correspondence of the CS damper location. (d) Acceleration of the rotor on the mock-up impeller. (e) Relative displacement between rotor and stator in the lower part of the impeller mock-up. (f) Relative displacement between rotor and stator in the higher part of the impeller mock-up. 
(C) The last test is performed with the pump in rotation measuring the unbalance response in correspondence of the higher damper location. The experimental (solid line) and numerical results (dashed line) are reported in Figure 12. The critical speeds occur at $5000 \mathrm{rpm}$ and 25,000 $\mathrm{rpm}$. The damping action of the supports is effective at these velocities since they are both working in the transition region (see Figure 8), as expected. The good correspondence between the numerical and experimental results is a validation of the proposed methodology and modeling approach at component and system level.

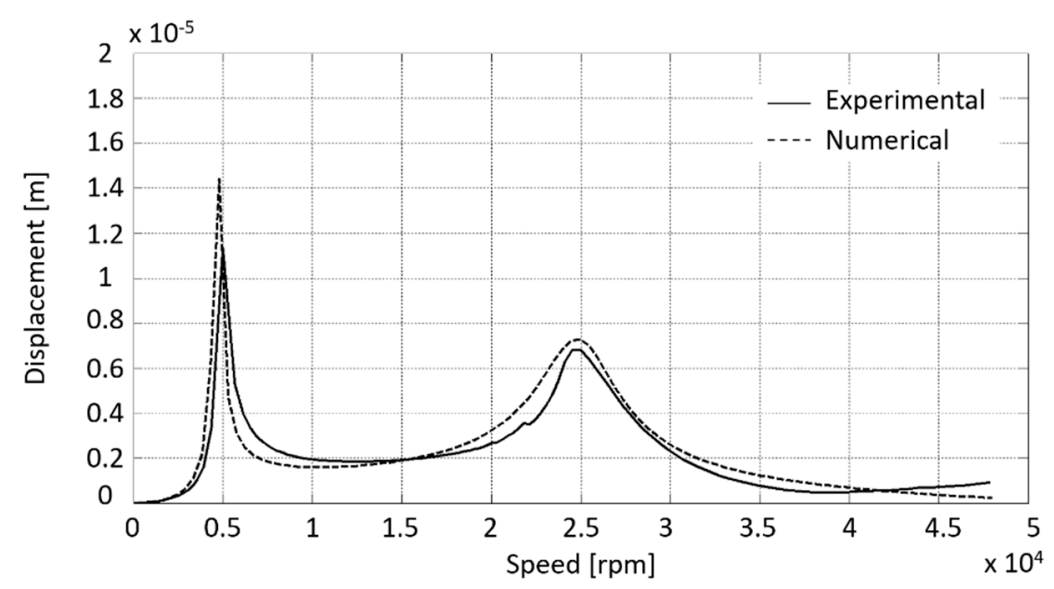

Figure 12. Characterization of the assembled pump in rotation. Unbalance response measured from $0 \mathrm{rpm}$ up to nominal speed $(48,000 \mathrm{Rpm})$ with capacitive displacement sensor placed in correspondence of the higher damper location.

\section{Conclusions}

In this paper, a methodology for the modeling, the testing, and the identification at the component level and at the system level of viscoelastic dampers for rotors has been illustrated. The tested dampers are realized with a viscoelastic element that is molded between metallic supports. The contribution in terms of damping and stiffness of the dampers has been evaluated by means of a dedicated machine realized to simulate the unbalance excitation on the supports. The parameters of the reference model of the dampers have been identified on the base of the experimental measurements by a Genetic Algorithm aiming to search the optimal values that minimize the difference between the numerical and the experimental results. Afterwards, the tested dampers have been installed in a turbo-molecular pump to validate the procedure also at system level. The pump has been tested at null speed by means of impact tests and in rotation by measuring the unbalance response. The validation of the proposed methodology has been conducted by comparing the experimental results with the numerical computations obtained by a model containing the rotor and the stator connected through the identified damper dynamics.

Author Contributions: All the authors developed the theoretical concepts and designed the experiments. Angelo Bonfitto implemented the simulations. Angelo Bonfitto and Andrea Tonoli wrote the theoretical sections. Angelo Bonfitto and Nicola Amati wrote the experimental sections. All the authors contributed (wrote, reviewed and supervised) to the final manuscript.

Conflicts of Interest: The authors declare no conflict of interest.

\section{References}

1. Ahn, Y.K.; Yang, B.S.; Morishita, S. Directional Controllable Squeeze Film Damper Using Electro-Rheological Fluid. ASME J. Vib. Acoust. 2002, 124, 105-109. [CrossRef]

2. Vance, J.M.; Ying, D. Experimental Measurements of Actively Controlled Bearing Damping with an Electrorheological fluid. ASME J. Eng. Gas Turbines Power 2000, 122, 337-344. [CrossRef] 
3. Bleuler, H.; Cole, M.; Keogh, P.; Larsonneur, R.; Maslen, E.; Nordmann, R.; Okada, Y.; Schweitzer, G.; Traxler, A. Magnetic Bearings Theory, Design, and Application to Rotating Machinery; Springer: Berlin/Heidelberg, Germany, 2009.

4. Tonoli, A.; Amati, N.; Bonfitto, A.; Silvagni, M.; Staples, B.; Karpenko, E. Design of Electromagnetic Dampers for Aero-Engine Applications. J. Eng. Gas Turbines Power 2010, 132, 11. [CrossRef]

5. Chiba, A.; Fukao, T.; Ichikawa, O.; Oshima, M.; Takemoto, M.; Dorrell, D.G. Magnetic Bearings and Bearingless Drives; Elsevier: Oxford, UK, 2005.

6. Amati, N.; Silvagni, M.; Tonoli, A. Electromechanical Dampers for Vibration Control of Structures and Rotors. In Vibration Control; Lallart, M., Ed.; InTech: Rijeka, Croatia, 2010; Volume 1, p. 32. ISBN 9789533071176.

7. Vance, J.M. Rotordynamics of Turbomachinery; John Wiley and Sons: Hoboken, NJ, USA, 1988.

8. Yoshimura, N. Vacuum Technology, Practice for Scientific Instruments; Springer: New York, NY, USA, 2008.

9. Hoffman, D.M.; Singh, B.; Thomas, J.H., III. Handbook of Vacuum Science and Technology; Academic Press: Cambridge, MA, USA, 1998.

10. Tonoli, A. Dynamic characteristics of eddy current dampers and couplers. J. Sound Vib. 2006, 301, 576-591. [CrossRef]

11. Goldberg, D.E. Genetic Algorithms in Search, Optimization and Machine Learning; Addison-Wesley Publishing Co.: Boston, MA, USA, 1998.

12. Battaglia, G.J.; Maynard, J.M. Mean square Error: A Useful Tool for Statistical Process Management. AMP J. Technol. 1992, 2, 47-55.

13. Wang, Q.; Sopron, P.; Tracht, R. An Overview of Genetic Algorithms Applied to Control Engineering Problems. In Proceedings of the Second Conference on Machine Learning and Cybernetics, Xi'an, China, 2-5 November 2003.

14. Genta, G. Vibration Dynamics and Control; Springer: New York, NY, USA, 2009.

15. Moreira, R.A.S.; Corte-Real, J.D.; Rodrigues, J.D. A generalized frequency-temperature viscoelastic model. Shock Vib. 2010, 17, 407-418. [CrossRef]

16. Genta, G. Dynamics of Rotating Systems; Springer: New York, NY, USA, 2005.

(C) 2017 by the authors. Licensee MDPI, Basel, Switzerland. This article is an open access article distributed under the terms and conditions of the Creative Commons Attribution (CC BY) license (http:/ / creativecommons.org/licenses/by/4.0/). 